\title{
A CHAOTIC FUNCTION POSSESSING A SCRAMBLED SET WITH POSITIVE LEBESGUE MEASURE
}

\author{
I. KAN
}

\begin{abstract}
A continuous function, chaotic in the sense of $\mathrm{Li}$ and Yorke, is constructed which possesses a scrambled set of positive Lebesgue measure.
\end{abstract}

Introduction. A continuous function $f: I \rightarrow I$, where $I$ is a real finite interval, is called chaotic (in the sense of $\mathrm{Li}$ and Yorke [1]) provided there exists an uncountable set $S \subset I$ such that for any $x, y \in S, x \neq y$, and $p$ any periodic point of $f$ :

$$
\begin{aligned}
& \limsup _{n \rightarrow \infty}\left|f^{n}(x)-f^{n}(y)\right|>0, \\
& \liminf _{n \rightarrow \infty}\left|f^{n}(x)-f^{n}(y)\right|=0, \\
& \limsup _{n \rightarrow \infty}\left|f^{n}(x)-f^{n}(p)\right|>0,
\end{aligned}
$$

where $f^{n}$ is the $n$th iterate of $f$. We call any such set $S$ a scrambled set of $f$. We call a scrambled set $E$ extremally scrambled, iff for any $x, y \in E, x \neq y$, and $p$ any periodic point of $f$ :

$$
\begin{gathered}
\limsup _{n \rightarrow \infty}\left|f^{n}(x)-f^{n}(y)\right|=\operatorname{diam}(I), \\
\liminf _{n \rightarrow \infty}\left|f^{n}(x)-f^{n}(y)\right|=0, \\
\limsup _{n \rightarrow \infty}\left|f^{n}(x)-f^{n}(p)\right| \geq \operatorname{diam}(I) / 2 .
\end{gathered}
$$

J. Smítal has shown that $f:[0,1] \rightarrow[0,1]$ where

$$
f(x)= \begin{cases}2 x, & 0 \leq x \leq 1 / 2 \\ 2-2 x, & 1 / 2<x \leq 1\end{cases}
$$

has an extremally scrambled set of Lebesgue outer measure 1 [2]. This set is not Lebesgue measurable, and Smítal's construction of it makes use of the continuum hypothesis.

In the literature there is no known chaotic function possessing a scrambled set of positive Lebesgue measure. Here, without using the continuum hypothesis, we construct a continuous chaotic function $f:[0,1] \rightarrow[0,1]$, along with an extremally scrambled set $E$ of Lebesgue measure $\frac{1}{8}$.

Received by the editors August 8, 1983.

1980 Mathematics Subject Classification. Primary 26A18; Secondary 26A30, 54H20.

Key words and phrases. Chaos, scrambled sets. 
First we consider the class $\mathcal{G}$ of all continuous functions $g:[0,1] \rightarrow[0,1]$ such that $g(x)=3 x$ for $x \in\left[0, \frac{1}{3}\right]$ and $g(x)=3 x-2$ for $x \in\left[\frac{2}{3}, 1\right]$, and find a set $K \subset\left(0, \frac{1}{3}\right)$ which is an extremally scrambled set of every $g \in \mathcal{G}$. Any such $g$ is clearly chaotic. Then we construct a fat Cantor set $E \subset\left[\frac{3}{8}, \frac{5}{8}\right]$ with Lebesgue measure $\frac{1}{8}$. Finally we choose a particular $f \in \mathcal{G}$ which, when restricted to $E$, is a monomorphism (actually a homeomorphism) from $E$ to $K$. Since $K$ is an extremally scrambled set of $f, E$ is one also.

Preliminaries. Let $N$ denote the natural numbers. Let $\Omega=\{0,1\}^{N}$ be the space of all one-sided sequences of two symbols, along with the dictionary ordering relation $<$, and the topology of coordinatewise convergence. The shift $\sigma$ on $\Omega$ is defined by $(\sigma \omega)_{k}=\omega_{k+1}, k \in N$.

Let the map $\varphi: \Omega \rightarrow[0,1]$ be defined by

$$
\varphi(\omega)=2 \sum_{k=1}^{\infty} \omega_{k} 3^{-k}
$$

Then the image under $\varphi$ of $\Omega$ is the usual "middle thirds" Cantor set $C$, and $\varphi$ is an order preserving homeomorphism from $\Omega$ to $C$. Also, for any $g \in \mathcal{G}$, we have

$$
g(\varphi(\omega))=3 \varphi(\omega)-2 \omega_{1}=2 \sum_{k=1}^{\infty} \omega_{k+1} 3^{-k}=\varphi(\sigma \omega) .
$$

And, for $n \in N, g^{n}(\varphi(\omega))=\varphi\left(\sigma^{n} \omega\right)$.

The following simple lemma will prove useful.

LEMMA. (i) If $g \in \mathcal{G}, \alpha, \beta \in \Omega, \alpha_{n+j}=\beta_{n+j}$ for $j=1, \ldots, k$, then

$$
\left|g^{n}(\varphi(\alpha))-g^{n}(\varphi(\beta))\right| \leq 3^{-k} \text {. }
$$

(ii) If $g \in \mathcal{G}, \alpha \in \Omega, \alpha_{n+j}=0\left(\alpha_{n+j}=1\right)$, for $j=1, \ldots, k$, then

$$
g^{n}(\varphi(\alpha)) \leq 3^{-k}\left(g^{n}(\varphi(\alpha)) \geq 1-3^{-k}\right) .
$$

Proof. The lemma clearly follows from the fact that

$$
g^{n}(\varphi(\omega))=\varphi\left(\sigma^{n} \omega\right)=2 \sum_{j=1}^{\infty} \omega_{n+j} 3^{-j}
$$

I. We construct a set $K \subset C$, which is an extremally scrambled set of every $g \in \mathcal{G}$. The method used here is similar to that used by M. Osikawa and Y. Oono to construct a scrambled set $[3]$.

Let $r: N \rightarrow N$ be defined by

$$
r(k)=\inf \left\{l \in N \mid k \leq \sum_{j=1}^{l} j^{2}+2 j\right\}
$$

and let $s: N \rightarrow N$ be defined by

$$
s(k)=\sup \left\{l \in N \cup\{0\} \mid l+1 \leq\left(k-\sum_{j=1}^{r(k)-1} j^{2}+2 j\right) / r(k)\right\}
$$


where $\sum_{j=1}^{0} j^{2}+2 j$ is defined to be 0 . Then define $Z: \Omega \rightarrow \Omega$ by

$$
(Z(\omega))_{k}= \begin{cases}0 & \text { if } s(k)=0 \\ 1 & \text { if } s(k)=1 \\ \omega_{s(k)-1} & \text { if } s(k) \geq 2\end{cases}
$$

i.e., $Z(\omega)=01 \omega_{1} 0011 \omega_{1} \omega_{1} \omega_{2} \omega_{2} \cdots$. Let $K$ be the set $\varphi(Z(\Omega))$.

The map $Z: \Omega \rightarrow Z(\Omega)$ is an order preserving homeomorphism, and since $\varphi: \Omega \rightarrow$ $C$ is also, we see that $K$ is homeomorphic to $C$. This fact will be important in $\S$ III, where we construct a homeomorphism from the fat Cantor set $E$ of $\S I I$ to $K$.

PROPOSITION. $K$ is an extremally scrambled set of every $g \in \mathcal{G}$.

Proof. Let $x, y \in K, x<y$, and $p$ be any periodic point of $g$ with period $q$. It suffices to show that equations (4)-(6) hold with $g$ in place of $f$.

Let $\alpha=\varphi^{-1}(x)$ and $\beta=\varphi^{-1}(y)$. Then by the construction of $K$ we have, for any $k \in N$, infinitely many $n \in N$ such that $\alpha_{n+j}=\beta_{n+j}=0$ for $j=1, \ldots, k$. Thus, by (i) of the Lemma, (5) is satisfied. Since $x<y$, we also have infinitely many $n \in N$ such that $\alpha_{n+j}=0$ and $\beta_{n+j}=1$, for $j=1, \ldots, k$. Thus, by (ii) of the Lemma, (4) is satisfied. We also have infinitely many $n \in N$ such that $\alpha_{n+j}=0\left(\alpha_{n+j}=1\right)$ for $j=1, \ldots, k+q$ so that

$$
\begin{gathered}
\limsup _{n \rightarrow \infty}\left|g^{n}(x)-g^{n}(p)\right| \geq \max \left\{g^{n}(p) \mid n \in N\right\} \\
\left(\limsup _{n \rightarrow \infty}\left|g^{n}(x)-g^{n}(p)\right| \geq \max \left\{1-g^{n}(p) \mid n \in N\right\}\right)
\end{gathered}
$$

holds. Therefore,

$$
\limsup _{n \rightarrow \infty}\left|g^{n}(x)-g^{n}(p)\right| \geq 1 / 2
$$

holds.

II. Here we constructed a fat Cantor set $E \subset\left[\frac{3}{8}, \frac{5}{8}\right]$ so that $m(E)=\frac{1}{8}[\mathbf{4}, \mathbf{5}]$. The set $E$ will be the countable intersection of a nested sequence of compact sets $\left\{E_{n}\right\}$ where each $E_{n}$ is the union of $2^{n}$ disjoint closed intervals, exactly two of these intervals being contained in each one of the $2^{n-1}$ disjoint closed intervals of $E_{n-1}$. Also, the diameters of the intervals comprising $E_{n}$ go uniformly to 0 as $n$ goes to $\infty$.

Let $\Gamma_{n}=\{0,1\}^{n}$ denote the set of all two symbols of length $n$, and $\gamma_{k}$ is the $k$ th coordinate of $\gamma \in \Gamma_{n}$. There are $2^{n}$ elements of $\Gamma_{n}$.

The sine of $\pi / 6$ can be written as an infinite product $[6]$ as follows,

$$
\sin \frac{\pi}{6}=\frac{\pi}{6} \prod_{l=1}^{\infty}\left(1-\frac{1}{36 l^{2}}\right)=\frac{1}{2} .
$$

Let $E_{0}=\left[\frac{3}{8}, \frac{5}{8}\right]$ and let $E_{n}$ be the union of the $2^{n}$ disjoint closed intervals $I(\gamma)$, where $\gamma \in \Gamma_{n}$. The right-hand endpoint of $I(\gamma)$ is

$$
b(\gamma)=\frac{5}{8}-\frac{1}{4} \sum_{k=1}^{n} \gamma_{k} 2^{-k} \frac{\pi}{6} \prod_{l=1}^{6}\left(1-\frac{1}{36 l^{2}}\right)
$$


and the left-hand endpoint of $I(\gamma)$ is

$$
a(\gamma)=b(\gamma)-\frac{1}{4} 2^{-n} \frac{\pi}{6} \prod_{l=1}^{n+1}\left(1-\frac{1}{36 l^{2}}\right)
$$

It follows that if $n \geq 2, \gamma \in \Gamma_{n}, \lambda \in \Gamma_{n-1}$, then either $I(\gamma)$ and $I(\lambda)$ are disjoint, or $I(\gamma)$ is contained in $I(\lambda)$. The latter occurs iff $\gamma_{k}=\lambda_{k}$ for $k=1, \ldots, n-1$. The Lebesgue measure of $E_{n}$ is $\frac{1}{4} \frac{\pi}{6} \prod_{l=1}^{n+1}\left(1-1 / 36 l^{2}\right)$ and, since $E_{n} \downarrow E=\bigcap_{n} E_{n}$, we have $m(E)=\lim _{n \rightarrow \infty} E_{n}=\frac{1}{8}$.

A point is in $E$ iff it is a limit point of the right-hand endpoints of the intervals comprising the sets $E_{n}$. So, the map $\psi: \Omega \rightarrow E$ defined by

$$
\psi(\omega)=\frac{5}{8}-\frac{1}{4} \sum_{k=1}^{\infty} \omega_{k} 2^{-k} \frac{\pi}{6} \prod_{l=1}^{k}\left(1-\frac{1}{36 l^{2}}\right)
$$

is an order reversing homeomorphism.

III. We now choose a particular $f \in \mathcal{G}$ so that $f$ restricted to $E$ is a homeomorphism (monomorphism is all that is necessary) from $E$ to $K$. Then for any $x, y \in$ $E, x \neq y, p$ any periodic point of $f$, we have $f(x), f(y) \in K, f(x) \neq f(y), f(p)$ a periodic point of $f$. Thus $E$ will be an extremally scrambled set of $f$.

Let $f(t)=\varphi\left(Z\left(\psi^{-1}(t)\right)\right)$ for $t \in E$. Since $f \in \mathcal{G}$, we have $f(t)=3 t$ for $t \subset\left[0, \frac{1}{3}\right]$ and $f(t)=3 t-2$ for $t \subset\left[\frac{2}{3}, 1\right]$. We now define $f(t)$ for $t \subset E^{c} \cap\left(\frac{1}{3}, \frac{2}{3}\right)$ to be a linear interpolation of the values of $f$ on the nearest points of $E \cup\left\{\frac{1}{3}\right\} \cup\left\{\frac{2}{3}\right\}$ to $t$. More precisely, for $t \subset E^{c} \cap\left(\frac{1}{3}, \frac{2}{3}\right)$, we define

$$
f(t)=f\left(t_{l}\right)+\frac{\left(t-t_{l}\right)}{\left(t_{r}-t_{l}\right)}\left(f\left(t_{r}\right)-f\left(t_{l}\right)\right)
$$

where $t_{l}=\sup \left\{t^{\prime} \in E \mid t^{\prime}<t\right\} \cup\left\{\frac{1}{3}\right\}$ and $t_{r}=\inf \left\{t^{\prime} \in E \mid t^{\prime}>t\right\} \cup\left\{\frac{2}{3}\right\}$.

PROPOSITION. The function $f$ is continuous and $K$ is the homeomorphic image under $f$ of $E$.

PROOF. It suffices to show that $f$ is continuous, strictly decreasing on $\left[\frac{1}{3}, \frac{2}{3}\right]$, and $f(E)=K$.

The image under $f$ of $E$ is the image under $\varphi \circ Z$ of $\psi^{-1}(E)$, but $\psi^{-1}(E)=\Omega$, so

$$
f(E)=\varphi\left(Z\left(\psi^{-1}(E)\right)\right)=\varphi(Z(\Omega))=K .
$$

The function $f$ is continuous and strictly decreasing on $E \cup\left\{\frac{1}{3}\right\} \cup\left\{\frac{2}{3}\right\}$, since $f\left(\frac{1}{3}\right)=$ $1, f\left(\frac{2}{3}\right)=0, f(E)=K \subset\left(0, \frac{1}{3}\right)$, and also $\psi^{-1}$ is continuous and order reversing and both $\varphi$ and $Z$ are continuous and order preserving. On the rest of $\left[\frac{1}{3}, \frac{2}{3}\right], f$ is just contained by linear interpolation, so $f$ is continuous and strictly decreasing on $\left[\frac{1}{3}, \frac{2}{3}\right]$.

Conclusion. The $f$ constructed in the third part is a chaotic function possessing an extremally scrambled set $E$ of Lebesgue measure $\frac{1}{8}$.

REMARK. It is easy to find a chaotic function $h:[0,1]^{r} \rightarrow[0,1]^{r}$ which has a scrambled set of positive $r$ dimensional Lebesgue measure $m_{r}$. Let $h$ be defined by

$$
h\left(x_{1}, \ldots, x_{r}\right)=\left(f\left(x_{1}\right), \ldots, f\left(x_{r}\right)\right) .
$$


Then $E^{r}$ is a scrambled set of $h$ and $m_{r}\left(E^{r}\right)=\left(\frac{1}{8}\right)^{r}$. In fact, for any $x, y \in E^{r}, x \neq$ $y, p$ any periodic point of $h$, we have

$$
\limsup _{n \rightarrow \infty}\left|h^{n}(x)-h^{n}(y)\right| \geq 1
$$

$$
\liminf _{n \rightarrow \infty}\left|h^{n}(x)-h^{n}(y)\right|=0
$$

(9)

$$
\limsup _{n \rightarrow \infty}\left|h^{n}(x)-h^{n}(p)\right| \geq \sqrt{r} / 2 \text {. }
$$

\section{REFERENCES}

1. T. Y. Li and J. A. Yorke, Period three implies chaos, Amer. Math. Monthly 82 (1975), 985-992.

2. J. Smítal, A chaotic function with some extremal properties, Proc. Amer. Math. Soc. 87 (1983), 54-56.

3. M. Osikawa and Y. Oono, Chaos in $C^{0}$-endomorphism of interval, Publ. Res. Inst. Math. Sci. 17 (1981).

4. B. R. Gelbaum and J. M. H. Olmstead, Counterexamples in analysis, Holden-Day, San Francisco, Calif., 1964.

5. J. Kahane and R. Salem, Ensembles parfaits et séries trigonometriques, Actualités Sci. Indust., no. 1301, Hermann, Paris, 1963.

6. J. Conway, Functions of one complex variable, 2nd ed., Springer-Verlag, Berlin and New York, 1978, p. 174.

DEPARTMENT OF MATHEMATICS, UNIVERSITY OF ILLINOIS, URBANA, ILLINOIS 61801 